\title{
Adaptive Information Search: Age-Dependent Interactions between Cognitive Profiles and Strategies
}

\author{
Jessie Chin, Wai-Tat Fu, Thomas Kannampallil \\ University of Illinois at Urbana-Champaign \\ 405 N Mathews Avenue \\ Urbana, IL 61807 \\ \{chin5,wfu,tgk2\}@illinois.edu
}

\begin{abstract}
Previous research has shown that older adults performed worse in web search tasks, and attributed poorer performance to a decline in their cognitive abilities. We conducted a study involving younger and older adults to compare their web search behavior and performance in illdefined and well-defined information tasks using a health information website. In ill-defined tasks, only a general description about information needs was given, while in well-defined tasks, information needs as well as the specific target information were given. We found that older adults performed worse than younger adults in well-defined tasks, but the reverse was true in ill-defined tasks. Older adults compensated for their lower cognitive abilities by adopting a top-down knowledge-driven strategy to achieve the same level of performance in the ill-defined tasks. Indeed, path models showed that cognitive abilities, health literacy, and knowledge influenced search strategies adopted by older and younger adults. Design implications are also discussed.
\end{abstract}

\section{Author Keywords}

Age differences, web search, ill-defined task, search strategies, cost-benefit analysis, cognitive abilities, and health literacy

\section{ACM Classification Keywords}

H.3.3 Information Search and Retrieval: Search Process. I.2.8 Problem solving, control methods, and search. J.4 Social and behavioral sciences

\section{INTRODUCTION}

About 113 million internet users seek health information online and $50 \%$ of these users claim that search for healthrelated information has an impact on their health care routines [13]. $68 \%$ of the internet users over age sixty-five have used online health information to assist their medical decision making. But only $20 \%$ of the older population uses the web in their daily life. Previous studies have shown that the lower frequencies in the use of technology and the

Permission to make digital or hard copies of all or part of this work for personal or classroom use is granted without fee provided that copies are not made or distributed for profit or commercial advantage and that copies bear this notice and the full citation on the first page. To copy otherwise, or republish, to post on servers or to redistribute to lists, requires prior specific permission and/or a fee.

ACM CHI 2009, April 4-9, 2009, Boston, MA, USA.

Copyright 2009 ACM 978-1-60558-246-7/09/04 ...\$5.00 poorer search performance of older adults can be attributed to the degeneration of several fluid cognitive abilities, such as working memory, visual and spatial attention, and processing speed ([8], [6], [19])

Older age is a critical factor that determines success in web search due to the sophisticated information processing demands involving perceptual and cognitive functions [23]. Previous research [7] has suggested that the two most relevant factors that affect internet search behavior for older adults are: (a) worse manual motor skills (in terms of moving a mouse) and (b) the decline in working memory, which creates difficulty in keeping track of where they are in a highly dynamic information structure such as the internet.

Another important aspect of web search is the nature of the information task, i.e., whether the search problem is welldefined or ill-defined. Most previous research has focused on well-defined tasks, where the participants search for specific information, usually contained in a single web page or source. Ill-defined tasks are characterized by general information needs. Relevant information is distributed across multiple web pages and it is often the case that no single web page contains all the information required to complete the task. For example, consider that a user is trying to find information for a family member who has back pain, but does not know precisely what information is available or relevant. After an exploratory search on a medical website, several information items seem to be relevant; but, he cannot get a specific item that properly answers his questions. In this case, the user has to gather the information he thinks is valuable to his specific information needs. Thus, individuals may search differently according to their information needs and how they interpret the available information. Therefore, there is usually no single solution for these ill-defined problems.

Recently, many researchers have proposed the concept of E-Health [2], a movement that attempts to harness the large amount of health information available on the internet to support self-care and to facilitate patient's everyday healthrelated needs and decisions. Studies have evaluated web search behavior of older adults because they are most likely the consumers of health information. However, these studies tend to focus on information-seeking behavior in 

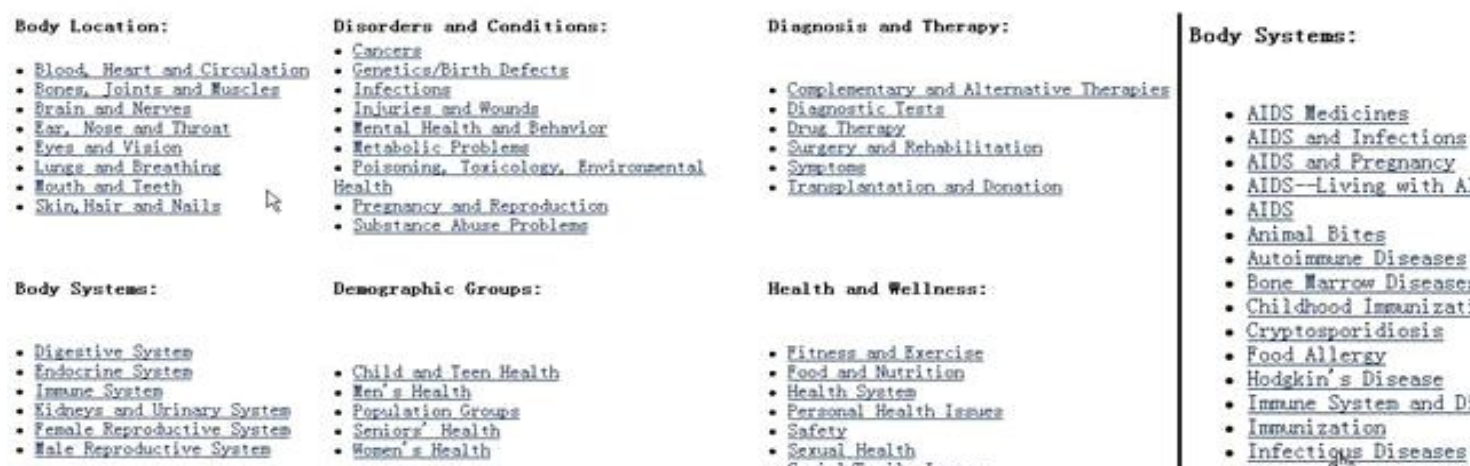

\section{Hagh Risk Pregancy}

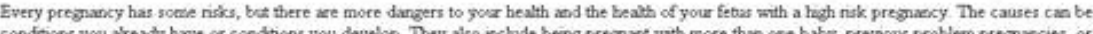

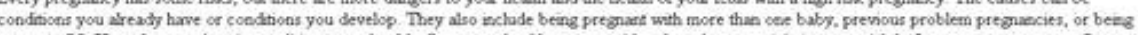

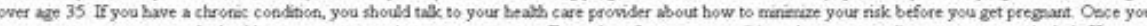

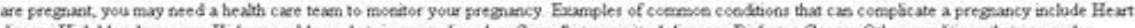

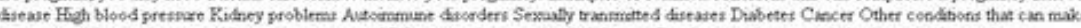

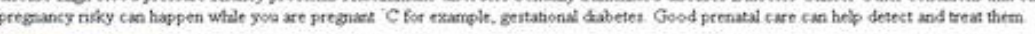

Figure 1. The three major page layouts of the website: (a) the main page showing all categories and subcategories of medical terms (top left), (b) the subcategory page showing all items within a subcategory that was selected on the main page (right), (c) the item page showing the description of the medical item that was selected on the subcategory page.

well-defined tasks, and found that older adults performed worse than younger adults in these tasks (for example, older adults could not find the necessary target information). One major motivation for our current study is to understand how older adults perform in the more general context of EHealth by analyzing web search behavior in ill-defined tasks, in which information-seeking is only one of the many cognitive activities. Comparisons between performance in ill-defined and well-defined tasks will shed light on how to promote and improve E-Health among older adults.

Some issues in web search are not yet well-understood. These include how age differences influence the adoption of search strategies, and how they in turn influence the search performance. We hypothesize that younger adults who have higher psychomotor speed can browse more numbers of web pages than older adults in a fixed period of time. Because of the lower cost to click a link, younger adults tend to prefer a more exploratory search strategy. In contrast, the exploratory search strategy may not be as costeffective for older adults, as there is a higher cost to each link selection due to their declining visual and motor skills.

Prior research often explained age differences in terms of correlations between cognitive abilities and search outcome. Instead, we investigate how adults in different age groups would use different search strategies in different information tasks (ill- or well-defined), and how these search strategies might mediate final search performance. A better understanding of the relationship among age, cognitive profiles, search strategies, and search performance would benefit designing and developing better health search technologies and interfaces, especially for older adults. We specifically explore the following research questions:

(a) How do the users' search strategies affect the performance in ill-defined and well-defined information search tasks?

(b) How do age differences, cognitive abilities, health literacy, and background knowledge affect the adoption of search strategies in different information search tasks (ill-defined and well-defined tasks)?

Studies exploring ill-defined exploratory tasks are limited. One notable exception is the recent work by Fairweather [11], who found that there are inherent qualitative differences in the manner of information search by older adults. Older adults follow different paths and focus on specific zones in a webpage as they pursue their information goal. We strongly believe that our results will provide deeper insights on the information search behavior of older adults.

\section{METHOD}

We investigated the age differences in health information search behavior and performance in well- and ill-defined tasks. We also investigated the extent to which differences in search behavior could be attributed to differences in general cognitive abilities, health literacy, and medical knowledge between younger and older adults. In the rest of this section, we describe our experimental methods, participants, tasks, measures, and the procedures that were used for this study. 


\section{Participants}

Sixty-nine individuals participated in our study. Of this forty-one were younger adults (15 males and 26 females) ranging in age from 18 to 35 years $(M=22.39$, s.d. $=3.69)$ and twenty-eight were older adults ( 9 males and 19 females) ranging in age from 60 to 83 years $(M=69.71$, s.d. $=5.76)$. All participants were recruited from a university community to participate in our study. Approximately half the older participants were between the age of 60 and 70 while the other half was between the age of 71 and 83. All participants had some online browsing experiences and basic computer skills. Almost all participants had completed at least some years of college, and there was no significant difference on the level of education between the younger and older adults.

\section{Experimental Design}

We used a between-subject design to examine the relationships among cognitive abilities, health literacy, medical knowledge, information search behavior and performance in the ill-defined and well-defined tasks. All participants started with a battery of standardized tasks that measured their cognitive abilities, health literacy, and medical knowledge. Half of the participants performed the ill-defined tasks while the other half worked on the welldefined tasks $(N=34 ; N=35)$. After completing the information tasks, participants performed a post-task for their medical knowledge.

\section{Measures of Cognitive Abilities, Health Literacy and Medical Knowledge}

Three measures were collected from participants to understand how age influences the search behavior and performance. First, basic cognitive measures such as the processing speed and the working memory capacity were collected using a set of 7 tasks (see appendix 1 for details for these tasks). These 7 tasks have been successfully used in many previous studies to explain how changes in cognitive abilities predict changes in a wide range of behavioral measures for older adults (e.g., see [18]).

Second, we collected health literacy measures that evaluated the general capabilities of comprehension of medical information by the standardized tests STOHFLA (Short Test of Functional Health Literacy in Adults) [1] and REALM (Rapid Estimate of Adult Literacy in Medicine) [9]. Health literacy is defined as " the degree to which individuals have the capacity to obtain, process, and understand basic health information and services needed to make appropriate health decisions" [17]. Both STOHFLA and REALM tests were shown to predict the general capabilities for people to understand and interpret a wide range of health-related concepts $([9,17])$. The cognitive ability and health literacy measures were collected before participants performed the information tasks.

We also measured participants' medical knowledge by asking them to rate the relatedness of 24 medical concepts randomly selected from the MedlinePlus.gov website (http://www.nlm.nih.gov/medlineplus/ healthtopics.html). These medical concepts ranged from disease, symptoms, body locations, body systems and treatment. Participants were asked to rate, from a scale of 1 (least related) to 9 (most related), all possible pairs of concepts randomly selected from this set of 24 concepts (for a total of 276 comparisons). We also conducted the same set of pairwise comparisons with a physician who has more than 10 years of professional practice. We then calculated the correlation on the raw similarity ratings of the pairwise comparisons between the participants and the physician. The correlation was used as a measure of how similar the medical knowledge structure of the participants was to that of the physician in terms of the mental distance of selected medical concepts. This measure of medical knowledge was conducted both before and after the information search task so that changes in knowledge structures during the task could also be assessed.

\section{Information search tasks}

Participants were asked to search for medical information using a website that was modeled on the public website MedlinePlus.gov. We did not perform any manipulation on our website to make it harder for participants to use the website (see Figure 1 for a screen shot of our website). We selected about 750 health knowledge items under six main categories: Body Locations, Body Systems, Disorders and Conditions, Demographic Groups, Diagnosis and Therapy, and Health and Wellness. Under each main category, there were five to nine subcategories. For example, under Body Location, one could find the subcategory Bones, Joints and Muscles among 8 other subcategories, and under this subcategory, one could find Arthritis among with 26 other items.

Half of the participants from each age group performed the well-defined information tasks and the other half performed the ill-defined information tasks. For both types of tasks, the contents of the website were the same, only the information tasks were different. For both tasks, participants were first shown the description of a task on a web page. Participants then clicked on "Begin" to go to the main page of the website, which showed the major categories as well as the subcategories of all medical terms.

Participants then clicked on any of the subcategory links (the main categories are not clickable) to go to the page listing all medical terms under that subcategory. They can also use the "Back" button to go back to the main page, or click on any of the medical terms to see a detailed description. Based on results from previous studies (e.g., [12]) as well as our pilot studies, the font size was set to 12 (with a screen resolution of $1024 \times 768$ ), with blue letters in white background to make sure older adults can easily read the text on all web pages. 


\begin{tabular}{|l|l|}
\hline Variables & \multicolumn{1}{|c|}{ Definitions } \\
\hline Age & $\begin{array}{l}\text { Younger adults (18-35 years), Older adults } \\
\text { (60-85 years) }\end{array}$ \\
\hline $\begin{array}{l}\text { Types of } \\
\text { tasks }\end{array}$ & Ill-defined, Well-defined \\
\hline $\begin{array}{l}\text { Cognitive } \\
\text { Profile }\end{array}$ & $\begin{array}{l}\text { Cognition (sum of 7 normalized scores of } \\
\text { cognitive tasks [See Appendix } 1]), \text { Health } \\
\text { literacy (STOHFLA), Medical knowledge }\end{array}$ \\
\hline $\begin{array}{l}\text { Search } \\
\text { Behavior }\end{array}$ & $\begin{array}{l}\text { Broadness, Processing time, Comprehension } \\
\text { time, Bottom-up search index }\end{array}$ \\
\hline $\begin{array}{l}\text { Search } \\
\text { Performance }\end{array}$ & $\begin{array}{l}\text { The accuracy rate of the ill-defined-task- } \\
\text { related medical test and the accuracy rate of } \\
\text { targets found in the well-defined task }\end{array}$ \\
\hline
\end{tabular}

Table 1. The summary of variables

\section{III-defined information task}

Most problems people confront on a day-to-day basis are ill-defined problems [21]. In other words, people often do not know precisely what information they are looking for, but only have a vague idea of the kind of information they need. We designed 4 scenarios in our ill-defined information task to understand participants' search behavior. The four task scenarios were

(a) Task 1: A person feels pain and stiffness in his/her body and has trouble moving around.

(b) Task 2: A person feels a burning, itching, and sometimes tingling sensation on his/her body.

(c) Task 3: A person just came back from a trip overseas and feels feverish and chilly.

(d) Task 4: A person gains weight suddenly, feels fatigue, and has difficulty dealing with cold temperatures

In each scenario, participants were given a brief description on some symptoms and were instructed to use the website to gather more information regarding these symptoms, such as what were their possible causes, and for each of these causes what were the possible treatments, how likely were each of these diseases were prevalent in different populations (older vs. younger, male vs. female, etc.), and any other related information. Participants could copy and paste any information from the website to a notepad provided to them. Participants had 15 minutes to browse the website for each of the 4 ill-defined tasks. Participants were also told that they would be given a medical knowledge test after four tasks. During the medical knowledge test participants were not allowed to refer to their saved notes.

The medical knowledge test consisted of 64 true/false-type questions and four open-ended questions designed for these four ill-defined scenarios. These questions were about all the possible solution items from the 4 tasks, and thus represented how much information they acquired and integrated across multiple web pages during the search. And the performance on this test should imply the degree they solved the ill-defined problems. Thus, the accuracy rate of the medical knowledge test was used as a measure of search performance in the ill-defined tasks.

\begin{tabular}{|c|c|c|}
\hline Measures & $\begin{array}{l}\text { Younger } \\
\text { Adults }\end{array}$ & $\begin{array}{l}\text { Older } \\
\text { Adults }\end{array}$ \\
\hline DSST Score & $46.08(5.76)$ & $29.93(7.75)$ \\
\hline $\begin{array}{l}\text { Letter Comparison Reaction } \\
\text { Time (ms) }\end{array}$ & $\begin{array}{l}1374.89 \\
(240.5)\end{array}$ & $\begin{array}{l}1946.64 \\
(272.5)\end{array}$ \\
\hline $\begin{array}{l}\text { Pattern Comparison } \\
\text { Reaction Time (ms) }\end{array}$ & $\begin{array}{l}1066.46 \\
(173.3)\end{array}$ & $\begin{array}{l}1366.84 \\
(219.6)\end{array}$ \\
\hline OSPAN Absolute Score & $45.44(18.3)$ & $\begin{array}{c}23.75 \\
(16.22)\end{array}$ \\
\hline $\begin{array}{l}\text { Flanker Test Reaction Time } \\
\text { (ms) }\end{array}$ & $\begin{array}{l}1014.50 \\
(170.3) \\
\end{array}$ & $\begin{array}{l}1395.02 \\
(235.6)\end{array}$ \\
\hline $\begin{array}{l}\text { Corsi Block Tapping Task } \\
\text { Score }\end{array}$ & $16.61(2.70)$ & $13.79(2.15)$ \\
\hline Letter Sets Test Score & $21.59(4.62)$ & $15.86(5.19)$ \\
\hline REALM & $64.17(2.53)$ & $65.11(1.12)$ \\
\hline STOHFLA & $35.46(1.12)$ & $34.18(2.51)$ \\
\hline Medical Knowledge & $0.413(0.13)$ & $0.443(0.24)$ \\
\hline
\end{tabular}

Table 2. The descriptive statistics of all cognitive, health literacy and knowledge measures

\section{Well-defined information search task}

To show how search behavior and performance may differ between well- and ill-defined tasks, we created 24 welldefined information search tasks, in which participants were given a specific medical term and its definition, and were asked to use our website to find a page that contained this target information. Participants were given 90 seconds to find the target item. If participants found the target page, a screen would tell them that the target information was found; if they could not find the information within 90 seconds, they were asked to stop their current task and proceed to the next search problem. And the success of finding the exact target information should imply they solved the well-defined problems. Thus, the accuracy rate of targets found was used to measure search performance in the well-defined tasks. See appendix 2 for the list of welldefined search target items.

\section{Procedure}

Each participant attended two sessions conducted on separate days. The primary reason for splitting the experiments into two days was to avoid participants' fatigue. On the first day, participants performed all tasks that measured cognitive abilities, health literacy, and medical knowledge. These tests lasted for about 2 hours. On the second day, participants performed the information tasks and the post-hoc medical knowledge pairwise comparison. The second session lasted for about 1.5 hours. All participants took the same standardized computer-based tasks with standardized instructions in a quiet room. Participants were paid $\$ 25$ for their participation.

\section{RESULTS}

Before performing the statistical analysis, outliers ( $>3$ s.d.) and missing data were deleted. On average less than $5 \%$ of the data was deleted. Owing to technical difficulties 
encountered during data collection, two participants from the well-defined task and one participant from the illdefined task were excluded from the analysis.

We focused on analyzing the relationship among the variables shown in Table 1. There were three major parts in our analysis. First, we tested whether there were effects of age differences in the cognitive profiles of the participants. Second, we tested how age differences and cognitive profiles influenced search performance in both ill-defined and well-defined tasks. Finally, we investigated how age differences and cognitive profiles influenced search behavior. Path analysis was conducted to test different causal models for each of these variables.

\section{Age Differences in the Cognitive Profiles}

\section{Principal Component Analysis on Cognitive Measures}

Table 2 presents the descriptive statistics of all the cognitive profile measures. We performed a principal component analysis (PCA) on the 7 cognitive measures and identified 2 major factors $(\lambda=5.0 \& 1.2$ respectively). But, there was high overlap between these factors. The factors explained $72 \%$ (56\% and $16 \%$ respectively) of the total variance. As expected, most cognitive measures loaded on the first factor, which included measures of short-term memory, working memory, processing speed and visual attention (cognitive abilities). Because the first factor explained most of the variance, we decided to merge all cognitive measures by aggregating the individual normalized scores to a single composite score, which we called "Cognition" from now on, to simplify the analysis (see Table 1, definitions under Cognitive Profile).

\section{Effect of Age Differences on Cognition and Health Literacy} Analysis of Variance (ANOVA) showed significant main effect of age on cognition where younger adults were significantly better than older adults $(F(1,64)=86.25$, $p<.001)$. There was also significant effect of age difference on the STOHFLA (a measure of health literacy) ( $F$ $(1,64)=7.57, p<.01)$, where younger adults performed significantly better than older adults. This indicated that younger adults had better general abilities for comprehension of health related materials. However, the REALM score, which measured verbal abilities for processing health-related concepts, did not show significant differences between the two age groups.

\section{Effect of Age Differences on Medical Knowledge}

Correlations from the pairwise comparisons between the participants and the medical expert were compared. This was performed between the age groups both before and after the information tasks. All correlation values were converted to z-scores using Fisher's z-transformation. ANOVA tests showed that there was no significant difference between age groups on their medical knowledge. Thus, all participants had similar levels of medical knowledge before they performed the information search task.
To summarize, we found significant differences between age groups on their cognition and the STOHFLA measures, but not on their REALM and medical knowledge measures. We therefore decided to use only the STOHFLA measure as the primary health literacy measure for the rest of this paper.

\section{Analysis on Search Performance}

We performed a median split and recoded all search behavior and search performance measures. In the illdefined tasks, search performance was measured by the accuracy rate of final medical tests given to the participants, while for the well-defined tasks search performance was measured by the accuracy rate of the target items that were found. Participants who scored higher than the median were coded as 2, while the others were coded as 1 . The purpose of the recoding was to make the comparison between the two types of tasks more meaningful [4].

We performed an analysis to investigate how different variables such as age, cognitive profiles, types of tasks, and search behavior would influence search performance. None of the 4-way or 3-way interactions were significant; we therefore conducted separate 2-way ANOVAs to test each of their effects on search performance. None of the main or interaction effects between age and the cognitive profile measures were significant, suggesting that the two age groups and the different cognitive profiles did not differ in their search performance. However, there were significant interactions between health literacy and types of task ( $F$ $(1,31)=7.37, p<0.05)$ and between cognition and types of task $(F(1,31)=3.91, \quad p<0.05)$. Participants with higher cognition and health literacy measures performed better only in the well-defined tasks, but not in the ill-defined tasks. Consistent with previous results, higher cognitive abilities and health literacy helped performance on the welldefined tasks. Interestingly, performance on ill-defined tasks did not depend on the level of cognition and health literacy. Also, there was no difference between the distributions of well-defined search performance and illdefined search performance in our study.

\section{Age and Types of Tasks on Search Performance}

ANOVA tests showed that there was no significant difference in the main effects of age and type of the tasks (ill- or well-defined). However, the interaction between age and type of tasks was significant $(F(1,61)=16.57, p<.001)$. The older adults performed better in the ill-defined tasks, while the younger adults performed better in the welldefined tasks. This is an intriguing finding, as it suggests that although older adults found fewer target items in the well-defined tasks, they were actually better in collecting, integrating, and comprehending health information on the website, as reflected by their better knowledge test scores in the ill-defined tasks $(F(1,31)=18.32, p<.001 ; t(31)=-.181$, $p=0.07$ ).

This is a novel finding that has not been reported before, as previous studies focused solely on well-defined tasks. To further understand this interaction effect, we compared how 


\begin{tabular}{|l|l|}
\hline \multicolumn{1}{|c|}{ Search Behavior } & \multicolumn{1}{c|}{ Definition } \\
\hline Broadness & $\begin{array}{l}\text { The sum of the unique number of } \\
\text { categories and subcategories } \\
\text { browsed in one problem }\end{array}$ \\
\hline Processing time & $\begin{array}{l}\text { Time spent on the decision of } \\
\text { each link under categories and } \\
\text { subcategories }\end{array}$ \\
\hline Comprehension time & Time spent on the item pages, \\
\hline $\begin{array}{l}\text { Bottom-up search } \\
\text { index }\end{array}$ & $\begin{array}{l}\text { The proportion of clicks in same- } \\
\text { categories and same- } \\
\text { subcategories }\end{array}$ \\
\hline
\end{tabular}

Table 3. The definitions of search behavior measures search behavior measures such as broadness, processing time and comprehension time were different between age groups and task types, and to what extent they explained the differences in search performance.

\section{Effect of Search Behavior and Types of Tasks on Search Performance}

Different measures of search behavior were collected from the participants. There were two basic descriptive search behavior variables: (1) Link selection, represented by the number of links selected (clicked) during each information search task, and (2) time measures such as the time spent in each problem, time spent on a page, etc.

For link selections, we first counted the number of unique categories and subcategories they belonged to. We called the sum of the unique number of categories and subcategories a measure of the broadness of the search strategy. A higher value of this measure indicated that more categories and subcategories were explored during the task. In addition, for each link selection, we first noted whether it was made in the main page or the subcategory page (Figure 1). We then calculated the number of times the link selection was made in the same category or subcategory as the previous link selection. This number basically measured how often participants followed the structure imposed by the interface as they searched, and therefore reflected the extent to which the search strategy was influenced by bottom-up features of the interface. We call it the bottom-up search index. We will defer the discussion of the results on this index in the subsection on path models.

For time measures, we extracted the time spent on every page before a participant clicked on a link (or the back button). We called this the processing time, which reflected the amount of time spent processing text on the main and subcategory pages, as well as the decision on which link to click on these pages. In addition, we measured the comprehension time as time spent on the item pages, which reflected how much time they spent on comprehending the medical terms. The median split was also conducted on all search behavior measures as mentioned. All definitions of search behavior measures are summarized in Table 3 .

Two-way ANOVA on search performance showed that there were significant interactions between the task types
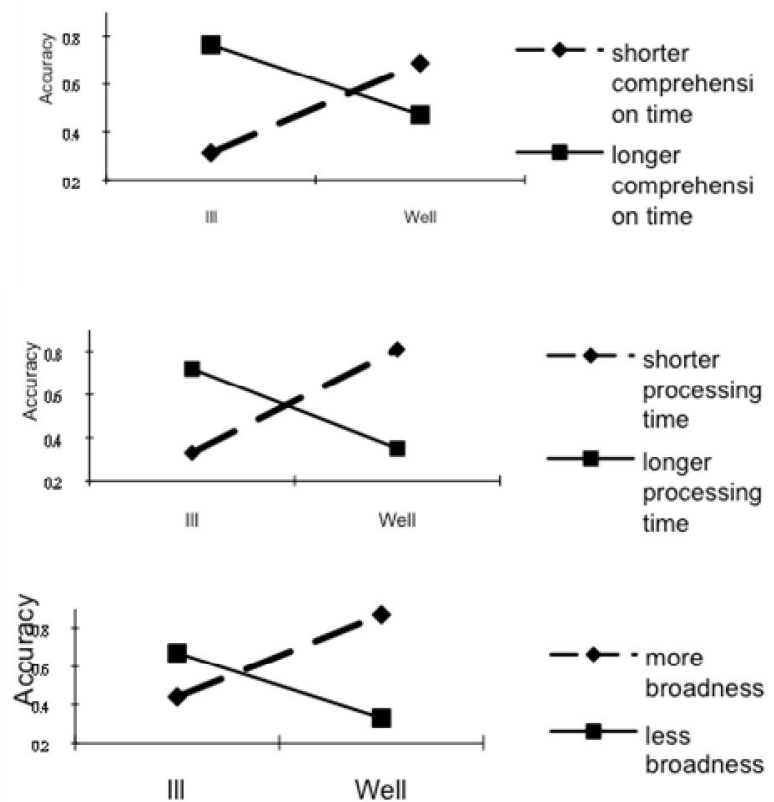

Figure 2. The interaction of the types of tasks and search strategies on search performance

(ill- vs. well-defined) and broadness $(F(1,61)=3.148$, $p<.05$ ). As shown in Figure 2 (bottom), participants who explored more categories of medical terms performed better only in the well-defined task. This implied that when the task was to look for specific information, exploring more categories of medical terms was more effective, as one could sample more information in a shorter period of time. However, for ill-defined information tasks that required deeper understanding and more semantic integration, broadness did not help.

The interaction between task types and processing time was significant $(F(1,61)=13.816, p<.05) \quad($ See Figure 2$)$. Participants with longer processing time performed better in the ill-defined tasks, but the reverse was true in the welldefined tasks. Finally, the interaction between task types and comprehension time was also significant ( $F=8.074$, $p<.05)$. Also, participants who spent more time reading, integrating, or elaborating the information performed better in the ill-defined task. In the well-defined task, the longer reading time resulted in inferior search performance.

The current pattern of results shows that participants who performed better in ill-defined and well-defined tasks adopted very different search strategies. For ill-defined tasks, participants who adopted a more focused search strategy, spent more time processing, deciding, and comprehending information performed better. On the contrary, for well-defined tasks, participants who adopted a more exploratory search strategy, leaving a web page and deciding on a click more quickly performed better. 


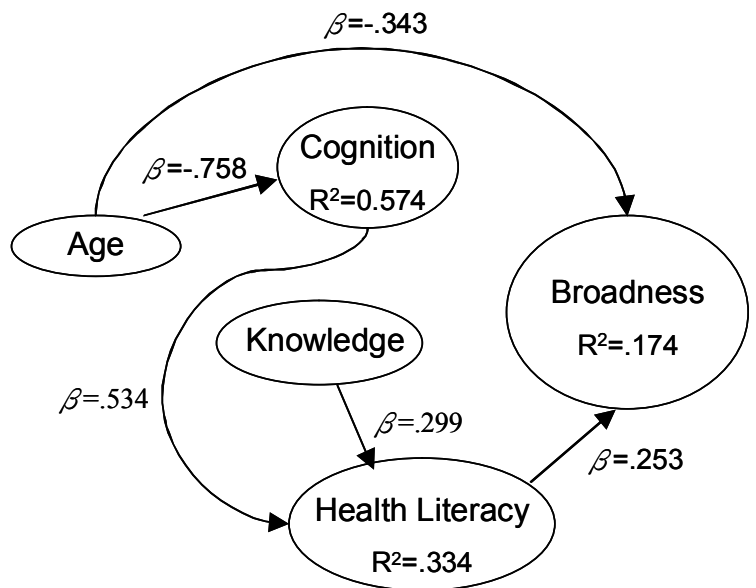

Figure 3. Path Model showing how age affects broadness along with cognition, knowledge, and health literacy

\section{Effect of Age and Search Behavior on Search Performance}

The 2-way ANOVA with age and each of the three search behavior variables (broadness, processing time, and comprehension time) on search performance showed that there was no main effect or interaction of age and search behavior. This result suggests that merely age and different search behavior cannot predict search performance in general; thus, we cannot define the "better search strategy" or "better information seeker" regardless of the types of tasks. Also, the 2-way ANOVA with age and types of tasks on three search behavior variables showed no interaction. This showed that older and younger adults adopted the same search strategies consistently across both ill-defined and well-defined tasks.

\section{Path Models: Effect of Age Differences on Search Behavior}

In the previous analyses, we showed how age differences were related to multiple variables and how they influenced search behavior and performance. To further understand age differences in the information tasks, we used hierarchical multiple regression to create path models for these variables. Because the ANOVA results showed there was no interaction between cognitive profiles and types of tasks on the search behavior, we merged the two types of tasks in the regression analysis to simplify the analysis, as the differences in cognitive profiles did not lead to different search behavior in ill-defined and well-defined tasks.

In our model, we set "Age" as the exogenous variable and the three search behavior measures, broadness, processing time, and comprehension time, as the dependent variables. We then added Cognition, Health Literacy, and Knowledge (medical knowledge) as mediating variables in the model. The results of the multiple hierarchical regressions are presented in Figure 3, 4 and 5 with each node listing the $\mathrm{r}^{2}$ for the stepwise regressions as well as the standardized beta coefficients for each link. Nodes without links in between indicate non-significant relations. For example, in Figure 3, the $r^{2}$ for regressing the variable "Age" on "Cognition" is
0.574, while that for regressing Cognition and Knowledge on Health Literacy simultaneously is 0.334 .

In all three models, age differences significantly predicted the decrease in fluid cognitive abilities. We also confirmed that age differences and health literacy are mediated by fluid cognitive abilities. Although there was no significant age difference in medical knowledge, we found medical knowledge predicted levels of health literacy in the model, which was consistent with previous research findings [14].

\section{First Model -- Broadness}

In the first model (see Figure 3), the variables accounted for $17.4 \%$ of the variance in broadness $(F(4,61)=3.208, p<.05)$. There was a direct path as well as an indirect path explaining how age predicted the broadness of search strategies. The indirect path suggested that age influenced the broadness of online search with the mediating effect of cognition and health literacy. There was also a moderating effect from medical knowledge through health literacy.

One could adopt a cost-benefit framework to explain this path model, which assumed that strategy selection was sensitive to the measure of information gain per unit cost (e.g., see [3]). For the younger adults, because of their faster processing speed and longer visual attention span, the cost of a broad search strategy - exploration of more categories and subcategories - was relatively lower than that for older adults. Therefore even if the average information gain (relevance of information) could be low (compared to a more focused search), a broad exploratory search strategy still resulted in a higher average information gain per unit cost and was therefore preferred by younger adults. Indeed, as we showed earlier, a broad search strategy did lead to better search performance on the welldefined task, in which the mere finding of the target items improved performance. On the other hand, for older adults, a more focused search based on top-down knowledge could increase the chance that each costly link selection would lead to useful information to maximize the average information gain per unit cost.

To test the hypothesis that younger adults were more exploratory, we conducted an ANOVA test to determine whether there was age difference effect on the bottom-up search index ( a measure of how often participants selected links that were in the same categories or subcategories). Results showed that younger adults did have a significantly higher bottom-up search index, indicating that they selected links within the same categories or subcategories consecutively more often than older adults $(F=19.214$, $p<.001)$. This result supported hypothesis that younger adults were exploratory, and their exploratory search strategies were apparently guided by the structural layout of the website (and thus more bottom-up).

\section{Second Model-Processing Time}

Variables in the second model (see Figure 4) accounted for $33.7 \%$ of the variance in processing time $(F(4,61)=7.758$, $p<.001)$. There were two indirect paths showing the effect 


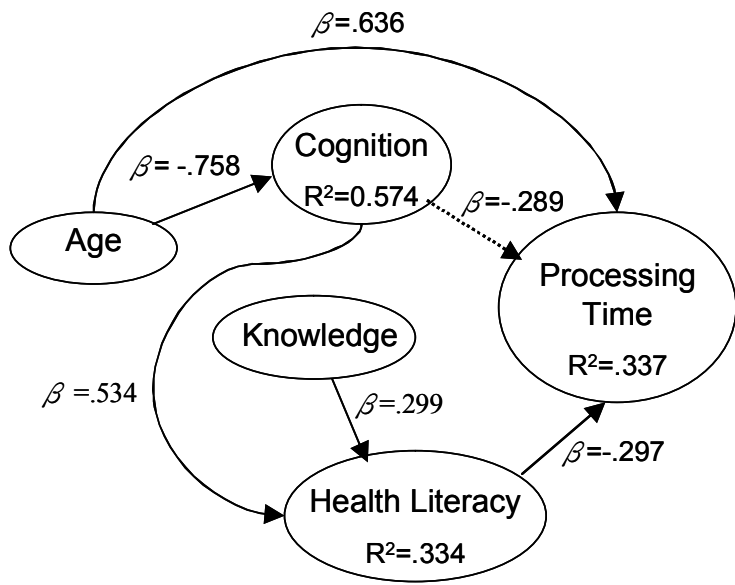

Figure 4. Path Model showing age affects processing time along with cognition, knowledge, and health literacy

of age difference on processing time. The first path was the same as that in the first model, which showed that age difference could be explained by the mediating effect of cognition and health literacy with a moderating effect from medical knowledge through health literacy. The second indirect path showed that longer processing time was predicted by lower fluid cognitive abilities. This suggested that older adults, with lower psychomotor speed and processing speed, performed tasks more slowly. Poorer visual attention and smaller working memory capacity also influence their decision process on staying or leaving a web page. The overall pattern of results was also consistent with the cost-benefit framework. The lower cognitive abilities suggested the link selection cost was higher, and therefore older adults spent more time to process the pages and were more careful in selecting links to avoid going back and forth between pages without acquiring useful information.

\section{Third Model-Comprehension Time}

Variables in the third model (see Figure 5) accounted for $15.7 \%$ of the variance in comprehension time ( $F$ $(4,61)=2.844 . p<.05)$. Similar to the first two models, age predicted cognition, which in turn predicted health literacy. However, unlike the first two models, health literacy did not predict comprehension time. Similar to the second model, there was an indirect path from age to cognition to comprehension time. However, unlike the first two models, medical knowledge predicted comprehension time. The results implied that older adults with poorer cognitive abilities spent more time to comprehending the description of medical terms. However, regardless of age, participants who had better medical knowledge spent less time comprehending the medical terms.

From the 3 path models we presented, we have identified how different search patterns between younger and older adults could be directly or indirectly predicted by their levels of cognitive abilities such as processing speed, health literacy, and medical knowledge. Younger adults who have better health literacy, quicker processing speed, better

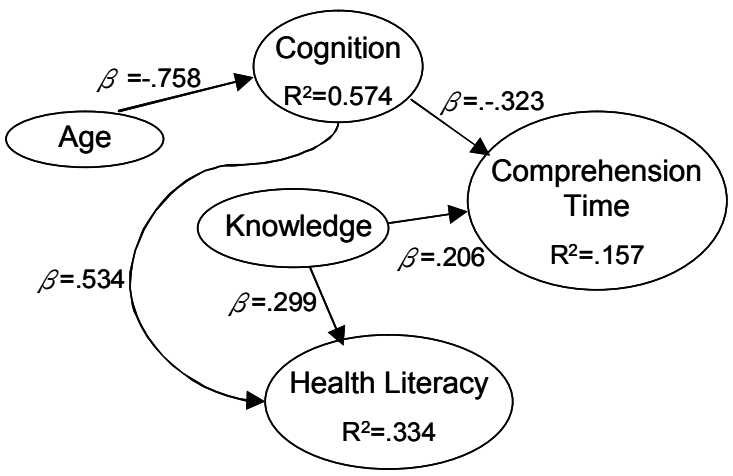

Figure 5. Path Model showing how age affects integration time along with cognition, knowledge, and health literacy

visual attention, and larger working memory capacity tended to adopt a bottom-up, exploratory search strategy. As a result, they tended to perform better in well-defined tasks. On the contrary, older adults scored lower on a wide range of cognitive tests and tended to use more focused search strategies, make decisions more carefully, and spend more time processing, comprehending and integrating information acquired from the website. As a result, for illdefined tasks, in which integration, interpretation, and comprehension of medical information were critical for performance, older adults performed better than younger adults.

The use of a more top-down, knowledge-driven strategy by the older adults was further supported by the finding that the medical knowledge of older adults became more similar to that of the expert after the information tasks, regardless of whether the task was ill-defined or well-defined. The more bottom-up, interface-driven search strategies adopted by younger adults did not lead to significant improvement in their medical knowledge, presumably because information acquired from the website was not actively integrated with their prior medical knowledge. In contrast, the top-down knowledge-driven strategies adopted by older adults would more naturally lead to integration between information acquired from the website with their prior medical knowledge. Indeed, research on reading comprehension suggested that active integration between reading materials and background knowledge is critical for a deeper understanding of textual materials [22].

To summarize, we found that older and younger adults were consistent in their choice of strategy across well and illdefined tasks. We performed a regression analysis to investigate how individual cognitive profiles constrained the selection of search strategies. The path models confirmed that different cognitive profiles due to aging influenced the adoption of search strategies. This in turn influenced search performance differentially in ill-defined and well-defined tasks. Consistent with the cost-benefit framework, older adults, who incur higher costs in clicking, scanning and memorizing search histories due to lower 
fluid cognitive abilities and health literacy, adopted a more focused, top-down search strategy.

\section{DISCUSSION}

Previous research on age differences in web search often concludes that older adults are generally not good at using the WWW to find information, and that their poorer performance can be explained by a decline in their general cognitive abilities [20]. Our study provides significant extensions and additions to prior research. First, in addition to testing and comparing the performance using welldefined information tasks in which participants were asked to search for specific target information, we developed a set of ill-defined information tasks. In these tasks, participants were only given a general topic to search for, and their performance was measured by the outcome of a set of broader cognitive abilities such as text comprehension and information integration, among which information search behavior served as a mediating variable. We found that although older adults performed worse in well-defined information tasks, they actually performed better than younger adults in ill-defined tasks. This is an exciting finding, as it suggests that older adults can perform just as well as younger adults when information search was studied as part of the activities that served the needs of higher-level cognitive goals. The result also becomes very relevant in the case of health care search tasks that, more often than not, are ill-defined in nature.

Second, we found that search performance depended on search strategies and the types of information tasks that participants were performing. Our results showed that for ill-defined information tasks, a top-down knowledge-driven search strategy was more effective in acquiring, integrating, and comprehending information on web pages; for welldefined information task, a bottom-up interface-driven search strategy was more effective in finding the target information. Strategy use was also well predicted by cognitive abilities, medical knowledge (background domain knowledge), and health literacy. Similar to most previous studies, our results showed that older adults tended to have lower general cognitive abilities and lower health literacy than younger adults, and these lower abilities predicted the use of the top-down search strategy. Apparently, older adults compensated their lower cognitive abilities by spending more time processing contents of the web pages to better comprehend the information, integrate with information read from previous pages and medical knowledge, and make better decisions on which links to select. Our results also showed that this top-down strategy was more than sufficient to allow older adults to effectively make use of the information on the website to serve their everyday information needs as in our ill-defined tasks. The current study focused on web search behavior for healthrelated information. There has been a growing number of research publications and discussions on how the internet may promote health care for the elderly by "empowering" them with accessible health-related knowledge and information that was previously not accessible (e.g., see [2]). Results from our study clearly indicate that studying information-seeking activities in isolation is not sufficient to understand how older adults comprehend and utilize medical information on the internet to make better healthrelated decisions or improve their self-care, or providing better health care for their relatives and friends. Clearly, information needs arising from these situations go beyond simple information retrieval. Future research should therefore focus on the complex interactions between information-seeking activities and other higher-level cognitive or even affective processes.

Results from our study also showed that, in addition to improving readability of materials on web pages, web design for the elderly should also consider how the semantic structure of the websites may facilitate top-down search strategies that we identified in our study, as well as how the site may facilitate higher-level cognitive activities such as information integration and comprehension. For example, we found that older adults spent more time deciding on which links to click and were more focused on a subset of the web pages while navigating. We believe that this could be because it was more difficult for older adults to keep track of the pages and the information contents that they have visited before and therefore there was a higher cost for them to explore different links on the website. It is therefore useful to provide some visualization tools for tracking their browsing activities. For example, a tree diagram, showing how the different web pages that they have visited are related to each other. This is particularly useful if the information content is inherently hierarchical in structure. A visualization tool will facilitate better information integration and decrease the cost of extra clicks as users navigate across multiple pages.

\section{REFERENCES}

1. Baker, D.W., M.V. Williams, R.M. Parker, J.A. Gazmararian, and J. Nurss, Development of a Brief Test to Measure Functional Health Literacy. Patient Education and Counseling, (1999), 38, 33-42.

2. Bass, S.B., How Will Internet Use Affect the Patient? A Review of Computer Network and Closed InternetBased System Studies and the Implications in Understanding How the Use of the Internet Affects Patient Populations. Journal of Health Psychology, (2003), 8, 25-38.

3. Brumby, D.P. and A. Howes, Strategies for Guiding Interactive Search: An Empirical Investigation into the Consequences of Label Relevance for Assessment and Selection. Human-Computer Interaction, (2008), 23, 146.

4. Cohen, J., P. Cohen, W. S., and L.S. Aiken, Applied Multiple Regression/Correlation Analysis for the Behavioral Sciences. 2003, New Jersey, NJ: Lawrence Erlbaum. 
5. Corsi, P.M., Human Memory and the Medial Temporal Region of the Brain. Dissertation Abstracts International, (1972), 34, 2.

6. Czaja, S.J., N. Charness, A. Fisk, C. Hertzog, S. Nari, W. Rogers, and J. Sharit, Factors Predicting the Use of Technology: Findings from the Center for Research Andeducation on Aging and Technology Enhancement (Create). Psych Aging, (2006), 21, 333-352.

7. Czaja, S.J. and C.C. Lee, The Internet and Older Adults: Design Challenges and Opportunities, in Communication, Technology and Aging, N. Charness, D.C. Parks, and B.A. Sabel, Editors. 2001, Springer: New York.

8. Czaja, S.J., J. Sharit, R. Ownby, D. Roth, and S. Nair, Examining Age Differences in Performance of a Complex Information Search and Retrieval Task. Psych Aging, (2001), 16, 564-579.

9. Davis, T.C., S.W. Long, R.H. Jackson, E.J. Mayeaux, and R.B. George, Rapid Estimate of Adult Literacy in Medicine: A Shortened Screening Instrument. Family Medicine, (1993), 25, 391-395.

10.Ekstrom, R.B., J.W. French, H.H. Harmon, and Dermen, Manual for the Kit of Factor-Referenced Cognitive Tests. 1976, Princeton, NJ: Educational Testing Service.

11. Fairweather, P. How Older and Younger Adults Differ in Their Approach to Problem Solving on a Complex Website. in Proceedings of the 10th international ACM SIGACCESS conference on Computers and accessibility. (2008). Halifax, Nova Scotia, Canada.

12.Fisk, A.D., W.A. Rogers, N. Charness, S.J. Czaja, and J. Sharit, Designing for Older Adults-Principles and Creative Human Factors Approaches. 2004, Washington, D. C.: CRC Press.

13.Fox, S. Online Health Search 2006. 2006 [cited 2008 September, 15].

14.Gazmararian, J.A., M.V. Williams, J. Peel, and D.W. Baker, Health Literacy and Knowledge of Chronic Disease. Patient education and counseling, (2003), 51, 267-275.

15.Lavie, N., Perceptual Load as a Necessary Condition for Selective Attention. Journal of Experimental Psychology: Human Perception and Performance, (1995), 21, 3 451-468.

16.McLeod, D.R., R.R. Griffiths, G.E. Bigelow, and J. Yingling, An Automated Version of the Digit Symbol Substitution Test (Dsst). Behavior Research Methods, Instruments, \& Computers, (1982), 14, 463-466.

17. Ratzan, S.C. and R.M. Parker, Introduction, in National Library of Medicine Current Bibliographies in Medicine: Health Literacy, C.R. Selden, et al., Editors. 2000, National Institute of Health, U. S. Department of Health and Human Services: Bethesda, MD.
18.Salthouse, T.A., Mediation of Adult Age Differences in Cognition by Reductions in Working Memory and Speed of Processing. Psychological Science, (1991), 2, 179-183.

19.Sharit, J., S. Czaja, M. Hernadez, Y. Yang, D. Perdomo, J. Lewis, C. Lee, and S. Nair, An Evaluation of Performance by Older Persons on a Simulated Telecommuting Task. Journal of Gerontology:Psychological Science, (2004), 59B, 305316.

20.Sharit, J., M. Hernandez, S. Czaja, and P. Pirolli, Investigating the Roles of Knowledge and Cognitive Abilities in Older Adult Information Seeking on the Web. ACM Transactions on Computer-Human Interaction, (2008), 15, 11-25.

21.Simon, H.A., The Structure of Ill-Structured Problems. Artificial Intelligence, (1973), 4, 181-204.

22.Stine-Morrow, E.A.L., L.M.S. Miller, D.D. Gagne, and C. Hertzog, Self-Regulated Reading in Adulthood. Psychology and Aging, (2008), 23, 1, 131-153.

23. Sutcliffe, A. and M. Ennis, Towards a Cognitive Theory of Information Retrieval. Interacting with computers, (1998), 10, 321-351.

24.Unsworth, N., R. Heitz, J. Schrock, and R. Engle, An Automated Version of the Operation Span Task. Behavior Research Methods, (2005), 37, 498-505.

Appendix 1. The seven cognitive measures we used were:

Visual motor speed was measured by the digit symbol substitution test (DSST;[16]) in which the coordination of visual perception and finger movement was the core component is this task. Processing speed was measured by the Letter Comparison and Pattern Comparison tasks [18]. Participants had to judge if the pair of two stimuli, such as two letter strings or two patterns, were the same or not as fast as they could. Working memory was measured by an automated version of the operation span task [24] which participants need to memorize a set of letters while solving some simple math questions alternatively. Corsi Block Tapping Task [5] required both visual search abilities and working memory to complete. Selective attention was measured by Flanker task [15] which manipulated perceptual load with adding different numbers of distracters with various distances from the target, participants had to respond as quickly as possible. Letter sets test was a timed paper test that measured inductive reasoning abilities [10].

\section{Appendix 2. Items used in the well-defined task}

Gout, Enterobiasis, Raynaud's disease, Cholesterol, Ehrlichiosis, Cryptosporidiosis, Klinefelter's syndrome, Shingles, Ergonomics, Diabetic kidney problems, Head and brain malformation, Breast cancer, Obesity, Nutrition for seniors, Genital warts, Snoring, Malaria, Disabilities, Patient rights, Electromagnetic fields, Critical care, Anesthesia, Domestic violence, Traveler's health. 